\title{
Phylogenetic Inference from Mitochondrial Genome Arrangement Data
}

\author{
Donald L. Simon and Bret Larget \\ Department of Mathematics/Computer Science \\ Duquesne University \\ Pittsburgh, PA 15282 \\ \{simon, larget\}@mathcs.duq.edu \\ http://www.mathcs.duq.edu/profs/\{simon.html, larget.html\}
}

\begin{abstract}
A fundamental problem in evolutionary biology is determining evolutionary relationships among different taxa. Genome arrangement data is potentially more informative than DNA sequence data in cases where alignment of DNA sequences is highly uncertain. We describe a Bayesian framework for phylogenetic inference from mitochondrial genome arrangement data that uses Markov chain Monte Carlo (MCMC) as the computational engine for inference. Our approach is to model mitochondrial data as a circular signed permutation which is subject to reversals. We calculate the likelihood of one arrangement mutating into another along a single branch by counting the number of possible sequences of reversals which transform the first to the second. We calculate the likelihood of the entire tree by augmenting the state space with the arrangements at the branching points of the tree. We use MCMC to update both the tree and the arrangement data at the branching points.
\end{abstract}

\section{Introduction}

Determining the evolutionary history and relationships among a group of taxa is a fundamental problem in evolutionary biology. Phylogenies are branching tree diagrams that display these evolutionary relationships. Phylogenetic inference may involve estimation of the true evolutionary relationships among a set of taxa, estimation of times of speciation, estimation of ancestral data, and the assessment of uncertainty in these estimates.

Swofford et al. 15. provides an excellent overview of commonly used methods for phylogenetic analysis from aligned DNA sequence data. More recently, several authors have developed a Bayesian approach to phylogenetic inference from DNA sequence data (Rannala and Yang [10; Yang and Rannala 16]; Mau, Newton, and Larget [8]; Larget and Simon [6]; Newton, Mau, and Larget [9]; Li, Pearl, and Doss [7]).

However, when the taxa of interest are quite distantly related, the difficulty in aligning sequences that have undergone substantial evolutionary change is great, and the sequences may no longer be phylogenetically informative. Several 
authors have argued that genome arrangement data is potentially more informative than DNA sequence data in comparing distantly related taxa, because large-scale genome rearrangements occur at much slower rates than nucleotide base substitution.

Mitochondrial genomes are circular and genes can appear on one of two strands. Nearly all animals have the same 37 mitochondrial genes. However, these genes are arranged differently in some species. These differences are a source of information to infer the evolutionary past.

Some recent papers which use genome arrangement data to make phylogenetic inferences include Smith et al.[11, Boore et al.[1], and Boore et al. 2]. The methods employed in these papers are not statistical.

Mitochondrial genome arrangement data is being collected at a rapidly increasing pace. In 1990, only about a dozen mitochondrial gene arrangements had been completely determined, but this number now exceeds one hundred (Boore [3]) and is expected to increase rapidly.

Despite this rapidly increasing amount of data, there is very little existing methodology for its use to reconstruct phylogenetic trees and to assess the uncertainty in the estimated evolutionary relationships. Most existing methodology is based on distance or parsimony methods. Sankoff et al. 12 is an early prominent example. In a recent article, Sankoff and Blanchette [13] describe a likelihood method for phylogenetic inference. This method, however, does not correspond to any particular mechanism for genome rearrangement.

This paper introduces a Bayesian framework for phylogenetic inference from genome arrangement data based on a likelihood model that assumes that reversals are the sole mechanism for genome rearrangement.

\section{A Model of Mitochondrial Genome Rearrangement}

A mitochondrial gene arrangement may be represented as a signed circular permutation where genes of the same sign are located on the same strand. There is a correspondence between signed circular permutations of size $g$ and ordinary signed permutations of size $g-1$ by choosing a single reference gene and a direction around the circle by which to list the remainder. There are several mechanisms through which mitochondrial genomes can rearrange. The 22 short (70-90 base pairs) genes code tRNAs. These short genes appear to rearrange by multiple mechanisms. The remaining 15 genes are longer (hundreds of base pairs). It is thought that inversions are the primary (and perhaps sole) mechanism by which large coding mitochondrial genes rearrange. For the remainder of this paper, we will ignore the 22 short tRNA genes and assume that reversals are the only mechanism of genome rearrangement. Given that an reversal occurs, our model assumes that all possible reversals are equally likely. 


\section{Representation and Modification of the Tree}

We represent a phylogeny of $s$ taxa with an unrooted tree topology $\tau$ and a $(2 s-3)$-vector of branch lengths $\mathbf{b}_{\tau}$. The branch lengths represent the expected number of reversals. The observed data at each leaf is a signed circular permutation of $g$ genes that we linearize and represent as a signed permutation of size $g-1$ by choosing a reference gene and direction around the circle to read the remaining genes. We represent the observed data at the leaf nodes with an array $L$ of $s$ signed permutations and augment our state space with an array $I$ of $s-2$ signed permutations at the internal nodes.

We describe three algorithms for proposing new trees. The first method randomly selects one of the $s-2$ internal nodes and performs a random number of reversals on its permutation. The number of reversals is chosen randomly by a geometric distribution with parameter $p=0.5$.

The second method is the LOCAL update method without the molecular clock used by BAMBE [6 14], an MCMC program for the Bayesian analysis of aligned nucleotide sequence data. We randomly pick one of the $2 s-3$ internal edges of the unrooted tree, designating its two nodes $u$ and $v$. The other two neighbors of $u$ are randomly labeled $a$ and $b$ and $v$ 's two other neighbors are randomly labeled $c$ and $d$ with equal probability. Set $m=\operatorname{dist}(a, c)$. Our proposal changes $m$ by multiplying edge lengths on the path from $a$ to $c$ by a random factor. We then detach either $u$ or $v$ with equal probability and reattach it along with its unchanged subtree to a point chosen uniformly at random on the path from $a$ to $c$. Specifically, $m^{*}=m \times e^{\lambda_{2}\left(U_{1}-0.5\right)}$ where $U_{1}$ is a uniform $(0,1)$ random variable and $\lambda_{2}$ is a tuning parameter. Let $x=\operatorname{dist}(a, u)$ and $y=\operatorname{dist}(a, v)$ be distances in the current tree. If $u$ is chosen to move, the proposal sets $x^{*}=U_{2} \times m^{*}$ and $y^{*}=y \times m^{*} / m$. If $v$ is chosen to move, $x^{*}=x \times m^{*} / m$ and $y^{*}=U_{2} \times m^{*}$. In both cases $U_{2}$ is a uniform $(0,1)$ random variable. If $x^{*}<y^{*}$, the tree topology does not change while $\operatorname{dist}\left(a, u^{*}\right)=x^{*}, \operatorname{dist}\left(u^{*}, v^{*}\right)=y^{*}-x^{*}$, and $\operatorname{dist}\left(v^{*}, c\right)=m^{*}-y^{*}$. If $x^{*}>y^{*}$, the tree topology does change as $u^{*}$ becomes a neighbor of $c$ and $v^{*}$ becomes a neighbor of $a$ while $\operatorname{dist}\left(a, v^{*}\right)=y^{*}, \operatorname{dist}\left(v^{*}, u^{*}\right)=x^{*}-y^{*}$, and $\operatorname{dist}\left(u^{*}, c\right)=m^{*}-x^{*}$. The Hastings ratio in this case is $\left(m^{*} / m\right)^{2}$.

The third method both deforms the tree using the LOCAL update method above and changes the permutation at one of the internal nodes. Consider the case when during the LOCAL update method the node $u$ is chosen to be moved. If the topology changes and the permutation at $u$ is either the same or one reversal away from the permutation at its old neighbor $a$, then the permutation at $u$ is copied from $c$, and a random reversal is applied with probability 0.5. If the topology does not change, or $u$ is more than one reversal away from $a$, then a random reversal is applied to $u$ 's permutation. In the other case, when the node $v$ is chosen to be moved, the same change is applied, except that the permutation may be copied from node $a$.

During one cycle the Markov chain procedure, each of the above three update methods is invoked in sequence. That is, the first method is used to change a node's permutation, then likelihood of the tree is calculated, and the new tree may be accept in place of the original tree. Then the LOCAL method without 
changing the permutation is invoked, the new tree is evaluated and possibly accepted. Finally, LOCAL with a permutation change is used, again possibly resulting in a tree being accepted.

\section{Calculation of the Likelihood}

The likelihood of a tree with augmented internal data is the product of the likelihoods for each branch of the tree times the probability of a particular arrangement at an arbitrary root node.

Calculation of the likelihood along a single branch. Let $p(x, y ; \beta)$ be the probability that gene arrangement $x$ is converted to gene arrangement $y$ after a Poisson $(\beta)$ distributed random number of equally likely reversals. This probability is:

$$
p(x, y ; \beta)=\sum_{k=d(x, y)}^{\infty} \frac{e^{-\beta} \beta^{k}}{k !} \times \frac{\#_{k}(x, y)}{\left(\begin{array}{l}
g \\
2
\end{array}\right)^{k}}
$$

where $d(x, y)$ is the length of a minimal sequence of reversals to convert $x$ to $y$ and $\#_{k}(x, y)$ is the number of different sequences of $k$ reversals that transform $x$ to $y$. Each term is the product of the Poisson probability of exactly $k$ reversals and the probability that a sequence of $k$ random reversals would transform arrangement $x$ to $y$.

We calculate the first three terms of this sum exactly and use the approximation

$$
\frac{\#_{k}(x, y)}{\left(\begin{array}{l}
g \\
2
\end{array}\right)^{k}} \approx \frac{1}{2^{g-1}(g-1) !}
$$

for the remaining terms. This approximation is based on the approximately uniform distribution of gene arrangements after a large number of random reversals from any starting gene arrangement. The resulting approximation is

$p(x, y ; \beta) \approx \sum_{k=d(x, y)}^{d(x, y)+2}\left(\frac{e^{-\beta} \beta^{k}}{k !} \times \frac{\#_{k}(x, y)}{\left(\begin{array}{l}g \\ 2\end{array}\right)^{k}}\right)+\left(1-\sum_{k=0}^{d(x, y)+2} \frac{e^{-\beta} \beta^{k}}{k !}\right) /\left(2^{g-1}(g-1) !\right)$

where $x$ and $y$ are the arrangements at the branch end-points, $\beta$ is the branch length, $d(x, y)$ is the length of the smallest sequence of reversals from $x$ to $y$ $\#_{k}(x, y)$ is the number of sequences of $k$ reversals that transform $x$ to $y$, and there are $g$ genes around the circle.

The likelihood of the data $(L, I)$ for a given tree $\left(\tau, \mathbf{b}_{\tau}\right)$ is

$$
p\left(L, I \mid \tau, \mathbf{b}_{\tau}\right)=\frac{1}{2^{g-1}(g-1) !} \times \prod_{i=1}^{2 s-3} p\left(x_{i}, y_{i} ; b_{i}\right)
$$

where $x_{i}$ and $y_{i}$ are the gene arrangements from $(L, I)$ that correspond to the end-points of branch $i$ and $b_{i}$ is the branch length of branch $i$. 
Prior distributions. We assume a uniform prior distribution over the discrete set of unrooted tree topologies and a uniform distribution over the set of possible gene arrangements at an arbitrary root node. We assume the vector of branch lengths is uniformly distributed over the positive orthant $\mathbf{R}^{2 s-3}$ subject to the constraint that the distance between any two leaves of the tree is less than a constant $T$. In the example below we use $T=16$.

Calculation details. There is a rapid algorithm for calculating $d(x, y)$ (Hannenhalli and Pevzner [4], Kaplan, Shamir, and Tarjan [5]). The difficult part of the calculation is determining $\#_{k}(x, y)$. Each of these counts corresponds to an equivalent count between an arrangement and the identity arrangement. By brute force calculation, we have determined these counts for $k \leq d(x, y)+2$ for $g \leq 10$. We take advantage of symmetries to reduce the necessary storage. The file that contains the three counts for all 4,674,977 possible arrangements up to symmetry for $g=10$ requires nearly $250 \mathrm{MB}$. To run our software on a data set with ten genes requires a computer with more than of $250 \mathrm{MB}$ of RAM to store and access the counts when needed. The time and space requirements to determine and store these counts increases exponentially. New approaches to quickly calculating or approximating these counts are necessary for the methods of this paper to be extended to gene arrangements with more genes.

\section{Example Data}

Due to the limitations of the current program, we tested the procedure on artificial data. We began with a known tree and a given signed permutation at the root. The permutation was of size 9 , simulating a circular signed permutation of size 10. The permutations at the other nodes were then generated in a depthfirst fashion by applying a number of random reversals to the parent of each node. The number of reversals was randomly chosen by a Poisson distribution with mean equal to the length of the branch to the parent. The data set given in Table 1 consists of the permutations at the leaves of the tree for one realization.

Table 1. Example Data Set

\begin{tabular}{cl}
\hline Taxon & Circular permutation \\
\hline a & $0,-5,-4,9,3,-8,2,7,-1,6$ \\
b & $0,-5,-4,9,3,1,-7,-2,8,6$ \\
c & $0,-7,-2,8,-3,-9,4,6,-1,5$ \\
d & $0,-6,-2,-1,-8,-7,9,3,4,5$ \\
e & $0,-5,-4,-3,-9,7,8,1,2,6$ \\
f & $0,7,-8,-6,4,1,5,-3,-2,9$
\end{tabular}

We completed four separate runs from randomly selected initial trees and obtained consistent results. Each run consisted of 10,000,000 cycles of which 
the first 100,000 were considered burn-in and discarded. Every tenth tree of the remaining data was sampled for inference. Each run took 90 minutes of CPU time on a $933 \mathrm{MHz}$ Pentium III running Redhat Linux 7.0.

The combined samples yield a posterior probability of 0.480 for the best tree topology which agreed with the true tree topology. The estimated Monte Carlo standard error of this probability based on the four independent samples was 0.025 .

Two other likely trees were generated by all four runs. The combined samples yield a posterior probability of 0.133 and 0.128 , respectively, for the two trees, with an estimated Monte Carlo standard error of 0.01 for each.

\section{Conclusions}

We have demonstrated a Bayesian approach towards genome arrangement data may be successful. The usefulness of this method depends on the solution or an approximation of a solution to the problem of finding the number of paths between two permutations. A brute force method of pre-calculating the distance works on this small example, but clearly is not practical for full mitochondrial genomes.

A second limitation is that if there are a large of number of reversals between taxa the posterior distribution will be spread over a large number of phylogenies. However, this would seem to be a limitation of the data rather than the method. It is unlikely that any method of phylogenetic reconstruction would be successful with data that is not very informative. In this instance, it would be useful to combine the genome arrangement data with other data such as nucleotide sequence data. This is simple to do within the MCMC paradigm.

In comparison to Bayesian methods for phylogenetic reconstruction from nucleotide sequence data, our current approach to Bayesian analysis of genome arrangement data requires longer simulation runs to achieve the same Monte Carlo standard error. In BAMBE 614, we were able to sum over all internal data whereas in this program such a summation would require enumerating all possible signed permutations which is far too large to be computed. Instead, we have data in the internal nodes which is updated at each cycle of the MCMC algorithm. This is not a limitation of this method, but one should be aware that longer runs are necessary.

Clearly this is a work in progress. We have only looked at small data sets in order to determine whether or not Bayesian methods can reconstruct phylogenies from genome arrangement data. We believe that with an appropriate approximation to the path counts this approach could be extended to the full mitochondrial genome and be computationally comparable to other methods which are not based on a likelihood model. 


\section{References}

1. Boore, J. L., T. M. Collins, D. Stanton, L. L. Daehler, and W. M. Brown. Deducing arthropod phylogeny from mitochondrial DNA rearrangements. Nature 376 (1995) 163-165

2. Boore, J., D.V. Lavrov, and W.M. Brown. Gene translocation links insects and crustaceans. Nature 392 (1998) 667-678

3. Boore, J. Mitochondrial gene arrangement source guide, version 5.0. DOE Joint Genome Institute. http://www.jgi.doe.gov/Mitochondrial_Genomics.html (2000)

4. Hannenhalli, S. and Pevzner, P. Transforming Men into Mice (polynomial algorithm for genomic distance problem). 36th Annual IEEE Symposium on Foundations of Computer Science (1995) 581-592

5. Kaplan, H., Shamir, R. and Tarjan, R.E.. Faster and Simpler Algorithm for Sorting Signed Permutations by Reversals. SIAM Journal on Computing 29(3) (1999) 880892

6. Larget, B. and Simon, D.L. Markov chain Monte Carlo algorithms for the Bayesian analysis of phylogenetic trees. Mol. Biol. Evol. 16 (1999) 750-759

7. Li, S., Doss H., Pearl D. Phylogenetic tree construction using Markov chain Monte Carlo. J. Amer. Stat. Assoc. 95 (2000) 493-508

8. Mau, B., M.A. Newton, and B. Larget. Bayesian phylogenetic inference via Markov chain Monte Carlo methods. Biometrics. 55 (1999) 1-12

9. Newton, M., B. Mau, and B. Larget. Markov chain Monte Carlo for the Bayesian analysis of evolutionary trees from aligned molecular sequences. In F. SeillierMoseiwitch (Ed.), Statistics in Molecular Biology and Genetics. IMS Lecture NotesMonograph Series, 33 (1999) 143-162

10. Rannala, B., and Z. Yang. Probability distribution of molecular evolutionary trees: A new method of phylogenetic inference. J. Mol. Evol. 43 (1996) 304-311

11. Smith, A., and Roberts, G.. Bayesian computation via the Gibbs sampler and related Markov chain Monte Carlo methods. J. R. Statist. Soc. B. 55 (1993) 3-23

12. Sankoff, D., Leduc, G., Antoine, N., Paquin, B., Lang, B.F. and Cedergren, R. Gene order comparisons for phylogenetic inference: evolution of the mitochondrial genome. Proceedings of the National Academy of Sciences 89 (1992) 6575-6579

13. Sankoff, D. and Blanchette, M. Phylogenetic invariants for Genome Rearrangement. J. of Comp. Biol. 6 (1999) 431-445

14. Simon, D. and B. Larget. Bayesian analysis in molecular biology and evolution (BAMBE), version 2.02 beta. Department of Mathematics and Computer Science, Duquesne University (2000)

15. Swofford, D.L., Olsen, G.J., Waddell, P.J., and Hillis, D.M. Phylogenetic inference. In D. M. Hillis, C. Moritz, and B. K. Mable, editors, Molecular Systematics, 2nd Edition. Sinauer Associates, Sunderland, Massachusetts (1996)

16. Yang, Z. and Rannala, B. Bayesian Phylogenetic Inference Using DNA Sequences: A Markov Chain Monte Carlo Method. Mol. Biol. Evol. 14 (1997) 717-724 